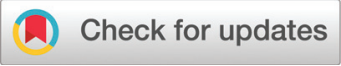

Cite this: Nanoscale, 2020, 12, 17880

\title{
Helical surface magnetization in nanowires: the role of chirality $\dagger$
}

\author{
Sandra Ruiz-Gómez, ${ }^{\text {a,b }}$ Claudia Fernández-González, ${ }^{c}$ Eduardo Martínez, ${ }^{d}$ \\ Victor Raposo, ${ }^{d}$ Andrea Sorrentino, ${ }^{b}$ Michael Foerster, ${ }^{b}$ Lucía Aballe, $^{\mathrm{b}}$ \\ Arantzazu Mascaraque, ${ }^{a, e}$ Salvador Ferrer ${ }^{b}$ and Lucas Pérez ${ }^{a, c, e}$
}

\begin{abstract}
Nanomagnetism is nowadays expanding into three dimensions, triggered by the discovery of new magnetic phenomena and their potential use in applications. This shift towards 3D structures should be accompanied by strategies and methodologies to map the tridimensional spin textures associated. We present here a combination of dichroic X-ray transmission microscopy at different angles and micromagnetic simulations allowing to determine the magnetic configuration of cylindrical nanowires. We have applied it to permalloy nanowires with equispaced chemical barriers that can act as pinning sites for domain walls. The magnetization at the core is longitudinal and generates at the surface of the wire helical magnetization. Different types of domain walls are found at the pinning sites, which respond differently to applied fields depending on the relative chirality of the adjacent domains.
\end{abstract}

Received 20th July 2020,

Accepted 17th August 2020

DOI: $10.1039 / \mathrm{d} 0 \mathrm{nr} 05424 \mathrm{k}$

rsc.li/nanoscale ducing magnetic tridimensional structures and enabling the appearance of new physics to exploit. These cylindrical nanowires can also be connected in 3D structures, enlarging the possibilities for the control of magnetic properties. ${ }^{19}$ Moreover, the particular spin texture observed in these cylindrical nanowires enables the movement of DWs without the limitation of the Walker Breakdown ${ }^{5,20}$ making NWs excellent candidates for their integration in devices based on domain wall movement.

In order to study this rich magnetic behavior as well as to exploit the properties of these cylindrical nanowires in applications, it is mandatory to have a description of the tridimensional magnetic configuration. In a recent work, ${ }^{21}$ using shadow photoemission electron microscopy with magnetic contrast (XMCD-PEEM), ${ }^{22,23}$ we reported the presence of three different types of DWs, one of them topologically protected under the application of magnetic fields in permalloy nanowires with chemical barriers. We also reported that such chemical barriers are effective pinning sites for DW. Our previous results with PEEM inferred the axial magnetizations of the wires from the absence of magnetic contrast in their core which was an indirect measurement that did not allow to determine their magnetization sense. Moreover, while the data evidenced that magnetization at the surface exhibited a circular pattern with upwards and downwards senses in each side of the wires in 2D images, the chirality of the surface magnetization was not determined. Here we present new results using $\mathrm{X}$ ray microscopy evidencing the sense of the axial magnetization as well as the chirality of the helical surface magnetization. We can thus unambiguously conclude that domain walls 
separating domains with opposite chirality are harder to move with magnetic field pulses than domain walls between adjacent domains with the same chirality, and we probe this rigidity upon field pulses of up to $750 \mathrm{mT}$.

\section{Methods}

We have electrodeposited permalloy NWs using similar conditions as described in our previous work. ${ }^{21}$ The NWs have a diameter of $250 \mathrm{~nm}$, with chemical barriers of $\mathrm{Fe}_{80} \mathrm{Ni}_{20}$, that are $20 \mathrm{~nm}$ in width and are separated $250 \mathrm{~nm}$ (see Fig. S1 from the ESI $\dagger$ ). The morphology of the NWs and the composition along the length of individual NWs were measured with a transmission electron microscope (TEM) JEOL JEM 2000FX.

XMCD-PEEM measurements were performed at the CIRCE beamline of the ALBA Synchrotron. ${ }^{24}$ Images at $\mathrm{Fe} \mathrm{L}_{3}$-edge were acquired with opposite photon helicity, and subtracted pixel by pixel in order to determine the in-plane magnetization component along the X-ray direction with nanometer resolution. Chemical contrast images were obtained as the difference in X-ray absorption at the $\mathrm{Fe}_{3}$ and $\mathrm{Ni} \mathrm{L}_{3}$ absorption edges. In all cases, images were acquired by collecting the distribution of secondary electrons at low kinetic energies.

TXM measurements were performed at the MISTRAL beamline of the ALBA Synchrotron, equipped with a transmission X ray microscope operating in the soft $\mathrm{X}$ ray range that utilizes photons extracted from a bending magnet source. ${ }^{25}$ A capillary condenser after the monochromator exit slit focuses the radiation to the sample, which is installed on a goniometer and on a $x-y-z$ translation stage. After the sample, a Fresnel zone plate with outermost zone width of $25 \mathrm{~nm}$ acts as objective lens of the microscope generating a $\sim \times 1200$ magnified image on a direct illumination CCD detector located at $2.5 \mathrm{~m}$ from the sample. The nanowires were deposited flat on top of a X-ray-transparent SiN membrane mounted in a sample holder installed at the microscope. The angle of the $\mathrm{X}$ ray beam with respect to the normal direction to the membrane could be varied by rotating the sample around a vertical axis, typically from $+55^{\circ}$ to $-55^{\circ}$.

In order to support our experimental observations, we have performed conventional micromagnetic simulations of the fabricated samples. Micromagnetics is a continuum theory based on the assumptions that (i) the modulus of the local magnetization vector is constant $\vec{M}(\vec{r})=M_{s} \hat{m}(\vec{r})$ with $\hat{m} \cdot \hat{m}=1\left(M_{\mathrm{s}}\right.$ being the saturation magnetization) and (ii) all vector quantities (the magnetization, the exchange and the self-magnetostatic fields, especially) vary slowly at the atomic scale. According to micromagnetic's theory, the effective field $\vec{H}_{\text {eff }}$ acting on the magnetization is the functional derivative of the energy density $\varepsilon$ with respect to the magnetization. Contributions to $\varepsilon$ arising from exchange, anisotropy, self-magnetostatic and applied field interactions are taken into account. In the present study we are only interested in equilibrium magnetic patterns, where the local magnetization is everywhere aligned to the local effective field, i.e. $\vec{m} \times \vec{H}_{\mathrm{eff}}=0$ (equilibrium equation). In order words, such equilibrium magnetic patterns correspond to minima of the energy system, $E=\int_{v} \varepsilon \mathrm{d} V$, where $V$ is the volume of the sample. For the present study only the exchange and the magnetostatic interaction are expected to contribute. The nanowire is numerically discretised by adopting a finite difference scheme. In order to do that, the continuous magnetization is sampled at a finite number of points in a mesh, so that the effective field and the equilibrium equation is converted into a finite set of simultaneous coupled equations, one for each mesh point. This set of non-linear coupled algebraic equations are solved simultaneously using iterative relaxations methods, such as underrelaxed Jacobi, steepest descent or conjugate gradient methods. Here the conjugate gradient method as implement in mumax $3^{26}$ is adopted. Further micromagnetic numerical details can be found in. ${ }^{27,28}$

\section{Analysis of magnetic contrast in TXM images}

Magnetic imaging at the X-ray microscope is based in extracting circular polarized photons from the bending magnet source and utilizing the angular sensitivity of magnetic dichroism which depends on the dot product of the unit photon spin $\hat{\sigma}$ and local magnetization $\hat{m}$ (unit vector). A magnetic film or wire of thickness $t$ illuminated with photons with energy tuned at a resonant absorption energy has a transmitted intensity $I$ (normalized to the incoming flux) given by:

$$
I=\exp (-\mu t)=\exp \left(-\mu_{0}(1+\delta \hat{m} \cdot \hat{\sigma})\right)
$$

where $\mu_{0}$ denotes the average linear absorption coefficient of a sample with zero average magnetization or with magnetization normal to the spin angular momentum $\sigma$ of the photons and $\delta$ is a factor which quantifies the magnitude of the magnetic dichroism. For X-rays tuned at the energies of the $\mathrm{L}_{3}$ and $\mathrm{L}_{2}$ absorptions of $\mathrm{Fe}$, the values are $\delta_{3}=0.28$ and $\delta_{2}=-0.18$, respectively. ${ }^{29}$ As indicated in (1) the absorption of $\mathrm{X}$ rays is enhanced or depressed depending on the sign of $\hat{m} \cdot \hat{\sigma}$ and of $\delta$. Considering absorption by Fe atoms, we denote by $\mu_{03}$ and $\mu_{02}$ the respective $\mathrm{L}_{3}$ and $\mathrm{L}_{2}$ absorption coefficients (which are related by $\mu_{03}=2 \mu_{02}$ ) and by $I_{3}$ and $I_{2}$ the transmitted intensities. Then,

$$
\left.\left.\Delta=\ln \left(-I_{3}\right)=2 \ln \left(I_{2}\right)=-\mu_{03} t\left(\delta_{3}-\delta_{3}\right) \hat{m} \cdot \hat{\sigma}\right)\right) .
$$

For a permalloy (Py) sample the absorption coefficient of $\mathrm{Ni}$ may be ignored since the magnetization of $\mathrm{Ni}$ is not resonantly probed by the X-ray beam and its absorption coefficient is about six times smaller than that of the Fe for thick samples. As permalloy has the stoichiometry $0.2 \mathrm{Fe} / 0.8 \mathrm{Ni}$, the inverse absorption length $\mu_{03}$ has to be multiplied by 0.2 . In addition, in our case we utilized left polarized circular polarization and $\hat{\sigma}=-\hat{k}$ (X-ray unit wavector). In order to obtain the magnetism of Fe in the NWs, we evaluated the quantity $\Delta$ in (2) in the $2 \mathrm{D}$ detector images which is proportional to $\hat{m} \cdot \vec{k}$ multiply by a 
positive constant. As a consequence, the chemical barriers of the nanowires that have a composition richer in $\mathrm{Fe}(0.8 \mathrm{Fe} / 0.2$ $\mathrm{Ni}$ ), will have larger $\Delta$ values, and thus will appear brighter in the micrographs, provided they have similar $\hat{m} \cdot \hat{k}$ as Py.

\section{Results and discussion}

\subsection{Magnetic microscopy}

In our previous work we reported the magnetic domain structure of permalloy nanowires with chemical barriers separated $1 \mu \mathrm{m}$. Using the technique of shadow analysis in XMCD-PEEM $^{30}$ we were able to establish the chemical barriers as effective pinning sites for DWs and also to identify different types of domain walls, one of them protected under the application of moderate magnetic fields. ${ }^{21}$ While separation of $1 \mu \mathrm{m}$ between notches provided interesting results, in view of future application in storage media as race track memories, it is desirable to decrease it. Consequently, we fabricated wires with chemical barriers separated $250 \mathrm{~nm}$ which make the measurements of the magnetization more demanding. In addition to shadow PEEM technique (see Fig. S2 $\dagger$ ), TXM and micromagnetic calculations allowed to obtain additional new information.

Fig. 1a sketches the geometry used to measure the TXM images, together with the definition of the rotation angle. Fig. $1 \mathrm{~b}$ and $\mathrm{d}$ show the magnetic images of the same NW taken at normal incidence $(\theta=0)$ and with two symmetrical angle rotations around the surface normal. The digits 1-7 indicate different grey sections separated by areas with bright white intensity which correspond to the chemical barriers. The image of Fig. $1 \mathrm{~b}$ at $\theta=0$ does not sense the axial magnetization since the wire is perpendicular to the incoming $\mathrm{X}$ ray beam and solely the magnetization having components along the $\mathrm{X}$ ray direction contributes to the image contrast. Segments 1, 2, 6 and 7 have all similar magnetic contrast: they are black at the top part indicating a magnetization that is antiparallel to $\vec{k}$, i.e., outwards, in this region. However in the bottom part of these segments the contrast is white (sign positive), which means that the magnetization is parallel and points inwards. Segment 4 has the same structure, but with the opposite sense of circulation. Segment 3 displays a diagonal line separating white and black areas. Segment 5 on the contrary has a different contrast distribution. It exhibits a sort of grey inclined strip that separates two domains with opposite circulation senses. As will be explained in detail later, segments 3 and 4 provide information on the helicity of the surface component of the magnetization.

Note that the visual appearance of the images at $\theta= \pm 25$ in Fig. 1c which are sensitive to the axial magnetization is similar to that of the image at $\theta=0$ which is not, indicating the relative importance of the surface magnetization in the measured contrast. In order to extract the axial magnetization, the magnetic images at 25 and -25 degrees were carefully aligned and subtracted: the projections of the longitudinal magnetization to the incoming X-ray wavector have opposite signs at positive and negative $\theta$ and they do not cancel upon subtraction whereas the magnetization components perpendicular to the wire cancel out since they have the same sign at $\theta$ and $-\theta$. The result of the subtraction is displayed in panel $\mathrm{d}$. The white/ black contrast scale in Fig. 1d has been enhanced relative to panels $\mathrm{b}$ and $\mathrm{c}$ for improved visualization since the contrast between axial domains is $70 \%$ lower than the contrast in Fig. 1b.

The overall contrast in Fig 1d may be divided in two groups: the left part of the image is darker than the right one i.e. seg- (a)
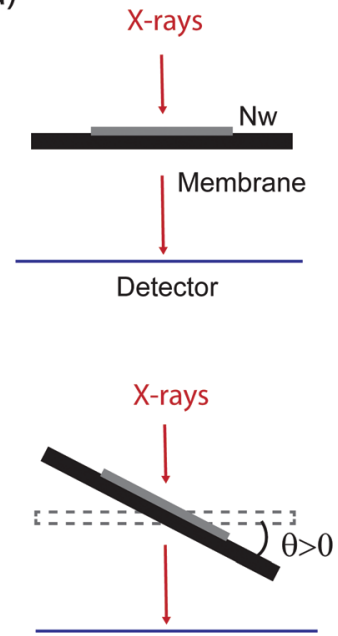

Detector (b)

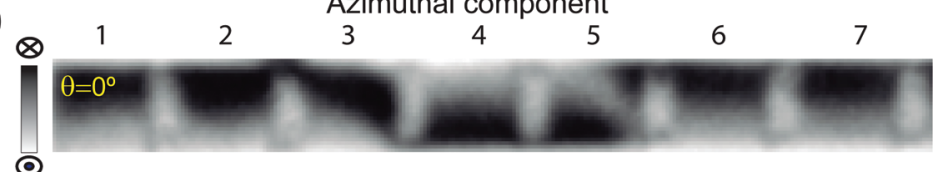

(c)
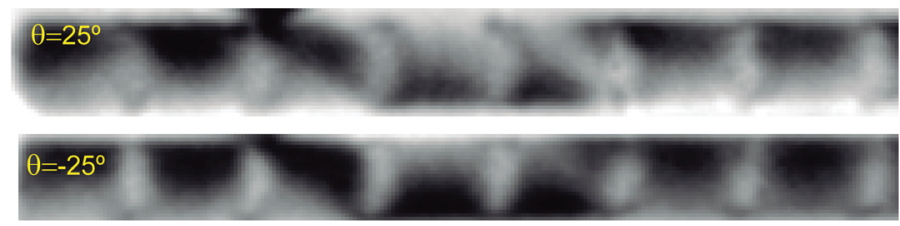

(d)

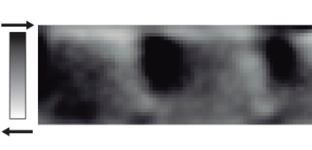

Axial component

(e)

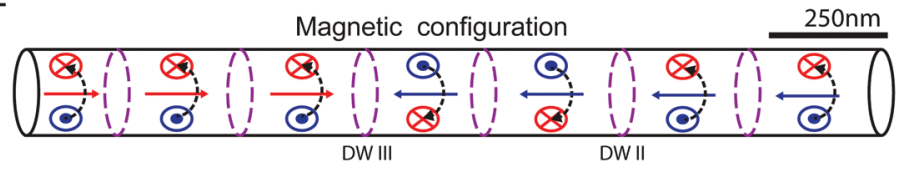

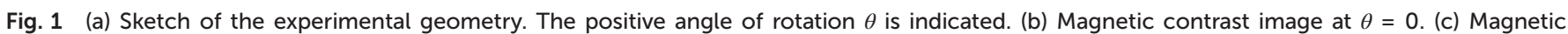

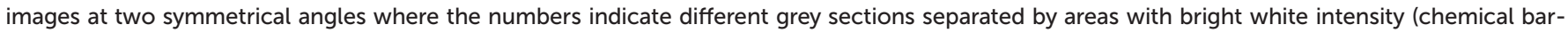
riers). (d) Subtraction of images in (b) to evidence the axial magnetization. (e) Sketch illustrating the magnetic configuration. 
ments 1,2 and 3 are darker than 4, 5, 6 and 7. This indicates two different orientations of the core magnetization: one common to $1-3$ and the other to $4-7$, which may be due to head-to-head or tail-to-tail configurations. The difference image also allows us to determine the relative orientations of the axial magnetizations, since head-to-head orientation results in white (black)contrasts in the left (right) sides of the image (Fig. 1d), whereas tail-to-tail would result in black (white) for left (right). The sketch in Fig. 1e indicates the orientations of the axial magnetizations: the magnetization in segment three points to the right (red arrows) and in segment four to the left (blue arrows). The figure also indicates the sense of the magnetization at the surface as inferred from Fig. 1b. As it will be discussed later, the peripheral magnetization has also a longitudinal component in the same sense that the axial one resulting in a helical magnetization with a defined chirality.

\subsection{Micromagnetic simulations}

We have performed micromagnetic simulations (using mumax $3 \operatorname{code}^{26}$ ) in order to describe the different magnetic configuration we have shown in the NWs. The diameter was set to $250 \mathrm{~nm}$ as well as the distance between chemical barriers. The length of the chemical barrier was kept constant at $20 \mathrm{~nm}$. Discretization size was chosen to be $4 \mathrm{~nm}$ for both layers. For both type of layers we consider a structure without magnetocrystalline anisotropy. The exchange constant was fixed at $A_{\text {ex }}=13 \times 10^{12} \mathrm{~J} \mathrm{~m}^{-1}$ and the damping constant at $\alpha=$ 0.02 . The saturation magnetization was set to $M_{\mathrm{S}}=0.8 \mathrm{MA} \mathrm{m}^{-1}$ in the NW and $M_{\mathrm{S}}=1.4 \mathrm{MA} \mathrm{m}^{-1}$ in the chemical barriers, expected values according to the composition of the NW and the chemical barrier. We used as initial conditions the configuration of magnetic domains giving rise to each DW obtained experimentally, relaxing afterwards the system to check the equilibrium magnetic configuration in each case.

In our previous work ${ }^{21}$ we proposed three types of magnetic configurations which are described in Fig. 2a: type I refers to a domain wall separating two head-to-head axial magnetizations and a homogeneous rotation sense, with circular magnetization lines in the outer part of the wire. In type II the axial magnetization has components pointing to the left in both domains but the surface magnetization exhibits helical field lines with opposite rotation senses (opposite chirality), the helixes at the right and left sides are right and left-handed respectively. Type III corresponds to opposite axial magnetizations and opposite senses of rotation of the surface magnetizations. Note that a $180^{\circ}$ rotation of one of the domains around a vertical axis in the figure makes both domains identical since the sense of the axial magnetization and that of the surface rotation are brought to coincidence. Consequently both domains in the figure have the same chirality but they are oriented head-to-head.

Panel b of Fig. 2 compares the measurements shown in Fig. 1b $(\theta=0)$ with the result of the simulations. The type II simulation has a good resemblance with the DW between segments 5 and 6 , since the light gray contrast along the diagonal in segment 5 and the dark grey contrast in segment 6 are well reproduced. Moreover, segments 5 and 6 in Fig. 1c display the same axial magnetization sense (see Fig. 1d) which confirms the DW to be type II. In contrast, the simulation of type III resembles the experimental image of segments 3 and 4 . Segment 3 in the type III simulation shows a non-uniform dark area as in the experiment. In Fig. 1d segments 3 and 4 have opposite head to head magnetizations and surface rotation senses in agreement with magnetic configuration type III. Note that the sense of advance of the surface helical mag- (a)

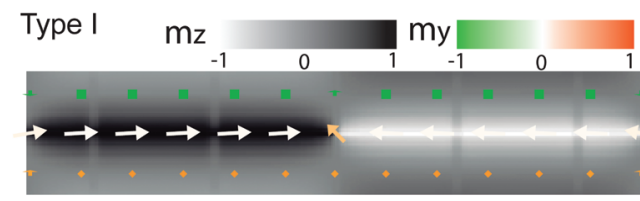

Type II

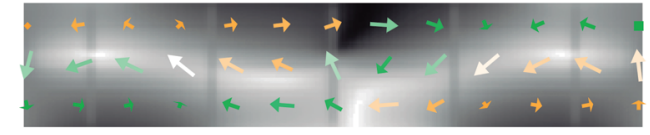

Type III

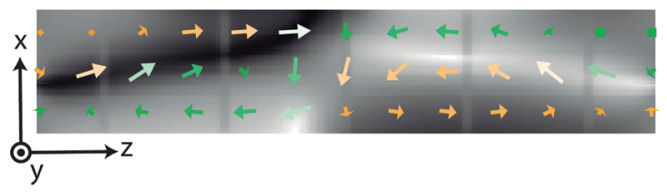

(b)

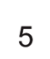

Type II
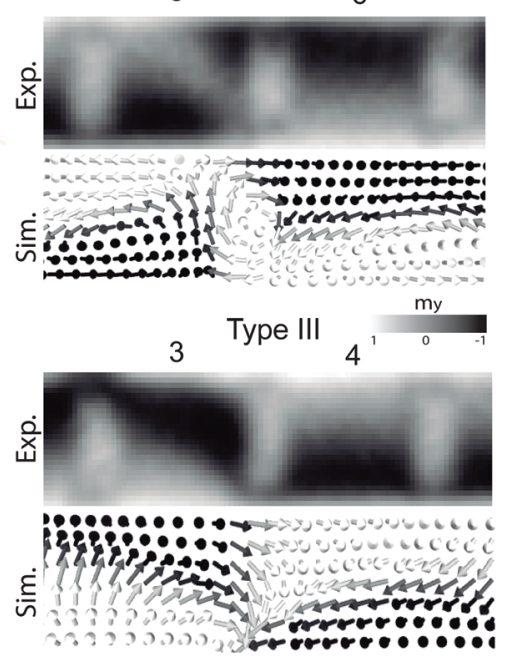

Fig. 2 (a) Final equilibrium state for the three different types of DWs obtained from micromagnetic simulations. (b) Comparison between micromagnetic simulations and TXM images. The numbers correspond with the one in Fig. $1 \mathrm{~b}$. 
(a) \begin{tabular}{llllllllll}
\multicolumn{1}{c}{ DW III DW III } & 2 & $\downarrow$ & 3 & $\downarrow$ & 4 & 5 & 6 & 7 & \multicolumn{1}{c}{ DW II }
\end{tabular} Magnetic configuration $\left[\begin{array}{|llllllllllllll}\hline 0 & 1 & 0 & 1 & 8 & 1 & 0 & 1 & 0 & 1 & 0 & 1 & 0 & 1\end{array}\right.$

(b)

(c)

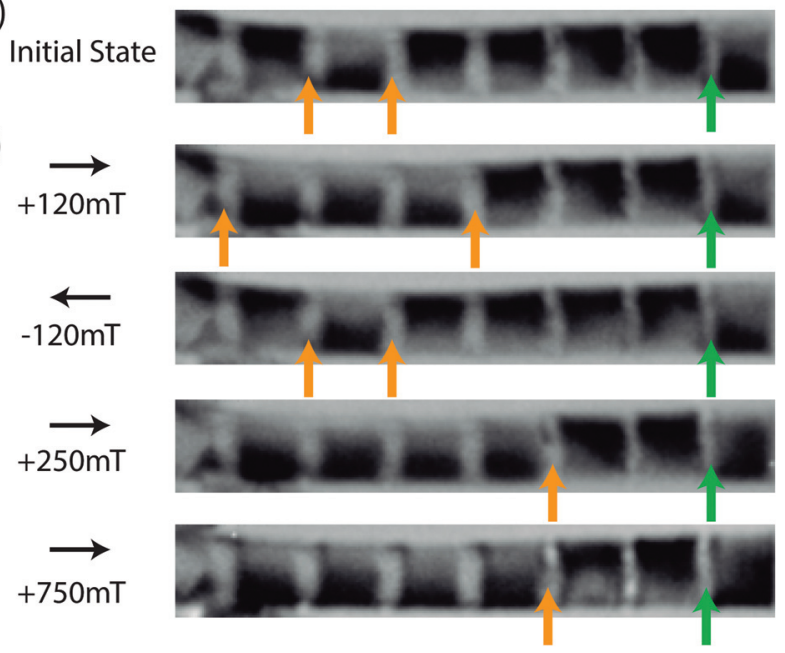

Fig. 3 (a) Schematics of the configuration of the magnetization of the initial state in panel (b). The application of magnetic field pulses produce the changes shown in panel (c) the two types of domains walls are marked with orange arrows (type III) and green arrows (type II).

netization is dictated by the sense of the axial magnetization via exchange coupling.

\subsection{Domain wall motion under the application of magnetic field}

We used a sample holder equipped with two small $\mathrm{Cu}$ coils which allowed to create pulses of horizontal magnetic fields, up to almost $\sim 1 \mathrm{~T}$ during about $15 \mu \mathrm{s}$. By selecting wires with horizontal orientation, the field was collinear with their axes and the motion of the domain walls could be investigated.

Fig. 3 shows selected results all of them at $\theta=0$ incidence. Panel a is a sketch of the initial configuration imaged in panel $\mathrm{b}$ in which the orientation of the magnetization of the different segments was determined. As indicated, there are two type III domains in the left part (orange vertical arrows) and one type II (green vertical arrow) at the right. The application of a pulse of $+120 \mathrm{mT}$ in the positive direction as shown in panel c (at the remanence) moves the orange arrows one segment in opposite senses as indicated. In more detail, the black contrast at the left side in segments 2 and 4 changes to the right side of the wire whereas segment 1 and 3 are unaltered. This indicates that the applied field changed the rotation sense of the surface magnetization of segments 2 and 4. This may be the consequence of a $180^{\circ}$ rotation of a whole domain around an axis normal to the wire which will keep its chirality unchanged while reversing the sense of the axial magnetization and the sense of the surface rotation. We adopt this interpretation as a reasonable working hypothesis. The application of a reversed field pulse of the same amplitude sets the magnetization as it initially was. Increasing the amplitude to $+250 \mathrm{mT}$ rotates again by $180^{\circ}$ segments 2 and 4 whereas segment 3 does not change. Then, the configuration of segments 2-3-4 becomes tail-to-tail in the axial magnetization and same chirality for all which means same sense of surface helical winding. A further increase to $+750 \mathrm{mT}$ does not produce additional changes, the DWs marked with an orange and green arrow are in the same position. However, note that segments $7-8$ which have the same axial magnetization pointing to the left and opposite chirality are not affected by the intense field pulse in spite the dipolar interaction that tends to orient their axial magnetization to the right. The difference between segments $2-3-4$ and $7-8$ is that in the first group they have the same chirality while that of 7 and 8 is opposite. This seems to indicate an important role of chirality in domain dynamics: domain walls separating segments of opposite chirality are hard to move.

\section{Conclusions}

We have demonstrated that the combination of TXM and micromagnetic simulations is a powerful tool for the determination of the magnetic configuration of nanowires. We investigated the magnetic configuration of magnetic domains and DWs of permalloy nanowires with Fe-rich chemical barriers as well as their behavior under the application of magnetic field pulses up to $750 \mathrm{mT}$. The nanowires have been found to have a core magnetized along their axis and a helical magnetization at the periphery. Exploiting the angular dependence of the magnetic dichroism allowed determining the sense of the core magnetizations of different parts of the wires as well as the chirality of the shell. Application of magnetic field pulses along the axis demonstrated the importance of the chirality in domain wall dynamics.

\section{Conflicts of interest}

There are no conflicts to declare.

\section{Acknowledgements}

This work is supported by the Spanish Ministry of Economy and Competitiveness (MINECO) through projects MAT201787072-C4-1-P, MAT2017-87072-C4-2-P and RTI2018-095303B-C53, the Comunidad de Madrid through Project NANOMAGCOST-CM P2018/NMT-4321 and by Consejería de Educación of Castilla y León through project SA299P18. IMDEA Nanociencia acknowledges support from the Severo Ochoa Programme for Centres of Excellence in R\&D (MINECO, Grant SEV-2016-0686). SRG acknowledges MINECO for FPI fellowship. 


\section{References}

1 S. S. P. Parkin, M. Hayashi and L. Thomas, Science, 2008, 320, 194.

2 D. A. Allwood, G. Xiong, C. C. Faulkner, D. Atkinson, D. Petit and R. P. Cowburn, Science, 2005, 309, 16881692.

3 R. Lavrijsen, J.-H. Lee, A. Fernandez-Pacheco, D. C. M. C. Petit, R. Mansell and R. P. Cowburn, Nature, 2013, 493, 647-650.

4 Z. Luo, A. Hrabec, T. P. Dao, G. Sala, S. Finicio, J. Feng, S. Mayr, J. Raabe, P. Gambardella and L. Heyderman, Nature, 2020, 579, 214.

5 M. Schobitz, A. D. Riz, S. Martin, S. Bochmann, C. Thirion, J. Vogel, M. Foerster, L. Aballe, T. O. Mente, A. Locatelli, F. Genuzio, S. L. Denmat, L. Cagnon, J. C. Toussaint, D. Gusakova, J. Bachmann and O. Fruchart, Phys. Rev. Lett., 2019, 123, 217201.

6 A. Fernández-Pacheco, R. Streubel, O. Fruchart, R. Hertel, P. Fischer and R. P. Cowburn, Nat. Commun., 2017, 8, 15756.

7 P. Fisher, D. Sanz-Hernández, R. Streubel and A. Fernández-Pacheco, APL Mater., 2020, 8, 010701.

8 S. D. Col, S. Jamet, N. Rougemaille, A. Locatelli, T. O. Mentes, B. S. Burgos, R. Afid, M. Darques, L. Cagnon, J. C. Toussaint and O. Fruchart, Phys. Rev. B: Condens. Matter Mater. Phys., 2014, 89, 180405.

9 Y. P. Ivanov, A. Chuvilin, S. Lopatin and J. Kosel, ACS Nano, 2016, 10, 5326-5332.

10 J. A. Fernández-Roldán, R. P. del Real, C. Bran, M. Vázquez and O. Chubykalo-Fesenko, Nanoscale, 2018, 10, 59235927.

11 W. Jiang, G. Chenc, K. Liu, J. Zang, S. G. te Velthuis and A. Hoffmann, Phys. Rep., 2017, 704, 1-49.

12 A. Fert, V. Cros and J. Sampaio, Nat. Nanotechnol., 2013, 8, 152.

13 C. Garg, S.-H. Yang, T. Phung, A. Pushp and S. S. P. Parkin, Sci. Adv., 2017, 3, e1602804.

14 C. Bran, E. Berganza, E. M. Palmero, J. A. FernandezRoldan, E. M. Palmero, J. Meier, E. Calle, M. Jaafar, M. Foerster, L. Aballe, A. Fraile-Rodriguez, R. P. del Real, A. Asenjo, O. Chubykalo-Fesenko and M. Vazquez, ACS Nano, 2018, 12, 5932-5939.

15 A. Nunez, L. Pérez, M. Abuín, J. P. Araujo and M. P. Proenca, J. Phys. D: Appl. Phys., 2017, 50, 155003.
16 M. S. Salem, P. Sergelius, R. M. Corona, J. Escrig, D. Görlitz and K. Nielsch, Nanoscale, 2013, 5, 3941.

17 C. Bran, E. Berganza, E. M. Palmero, J. A. FernándezRoldán, R. P. D. Real, L. Aballe, M. Foerster, A. Asenjo, A. F. Rodriguez and M. Vazquez, J. Mater. Chem. C, 2016, 4, 978-984.

18 S. Bochmann, D. Döhler, B. Trapp, M. Stano, O. Fruchart and J. Bachmann, J. Appl. Phys., 2018, 124, 163907.

19 A. Ruiz-Clavijo, S. Ruiz-Gómez, O. Caballero-Calero, L. Pérez and M. Martín-González, Phys. Status Solidi RRL, 2019, 13, 1900263.

20 M. Yan, A. Kakay, S. Gliga and R. Hertel, Phys. Rev. Lett., 2010, 104, 057201.

21 S. Ruiz-Gómez, M. Foerster, L. Aballe, M. P. Proenca, I. Lucas, J. L. Prieto, A. Mascaraque, J. de la Figuera, A. Quesada and L. Perez, Sci. Rep., 2018, 8, 16695.

22 S. Jamet, S. D. Col, N. Rougemaille, A. Wartelle, A. Locatelli, T. O. Mentes, B. S. Burgos, R. Afid, L. Cagnon, S. Bochmann, J. Bachmann, O. Fruchart and J. C. Toussaint, Phys. Rev. B: Condens. Matter Mater. Phys., 2015, 92, 144428.

23 A. Wartelle, J. Pablo-Navarro, M. Stano, S. Bochmann, S. Pairis, M. Rioult, C. Thirion, R. Belkhou, J. M. de Teresa, C. Magen and O. Fruchart, Nanotechnology, 2018, 29, 045704.

24 L. Aballe, M. Foerster, E. Pellegrin, J. Nicolas and S. Ferrer, J. Synchrotron Radiat., 2015, 22, 745-752.

25 A. Sorrentino, J. Nicolas, R. Valcarcel, F. J. Chichon, M. Rosanes, J. Avila, A. Tkachuk, J. Irwin, S. Ferrer and E. Pereiro, J. Synchrotron Radiat., 2015, 22, 1112.

26 A. Vansteenkiste, J. Leliaert, M. Dvornik, M. Helsen, F. Garcia-Sanchez and B. V. Waeyenberge, AIP Adv., 2014, 14, 107133.

27 L. Lopez-Diaz, D. Aurelio, L. Torres, E. Martinez, M. A. Hernandez-Lopez, J. Gomez, O. Alejos, M. Carpentieri, G. Finocchio and G. Consolo, J. Phys. D: Appl. Phys., 2012, 45, 323001.

28 O. Alejos, V. Raposo and E. Martinez, Mater. Sci. Technol., 2020, 015202-1.

29 J. Sthor and H. Siegmann, Magnetism From Fundamentals to Nanoscale Dynamics, Springer-Verlag, Berlin Heidelberg, 2006.

30 J. Kimling, F. Kronast, S. Martens, T. Bohnert, M. Martens, J. Herrero-Albillos, L. Tati-Bismaths, U. Merkt, K. Nielsch and G. Meier, Phys. Rev. B: Condens. Matter Mater. Phys., 2011, 84, 174406. 